\title{
Human Sebaceous Cis-6-Hexadecenoic Acid: Possible Application of an Innate Antimicrobial Substance to Cosmetic Products for Mucous Membrane
}

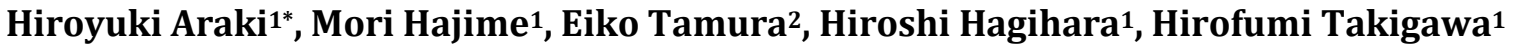 \\ ${ }^{1}$ Tochigi Research Laboratories, Kao Corporation, Tochigi, Japan \\ ${ }^{2}$ Odawara Research Laboratories, Kao Corporation, Kanagawa, Japan \\ Email: *araki.hiroyuki@kao.co.jp
}

How to cite this paper: Araki, H., Hajime, M., Tamura, E., Hagihara, H. and Takigawa, H. (2017) Human Sebaceous Cis-6-Hexadecenoic Acid: Possible Application of an Innate Antimicrobial Substance to Cosmetic Products for Mucous Membrane. Journal ot Cosmetics, Dermatological Sciences and Applications, 7, 133-147.

https://doi.org/10.4236/jcdsa.2017.72012

Received: March 11, 2017

Accepted: June 17, 2017

Published: June 20, 2017

Copyright $\odot 2017$ by authors and Scientific Research Publishing Inc. This work is licensed under the Creative Commons Attribution International License (CC BY 4.0).

http://creativecommons.org/licenses/by/4.0/

\begin{abstract}
The antimicrobial properties of cis-6-hexadecenoic acid (C16:1 $\Delta 6)$, a component of the innate human metabolome, were studied and its application to cosmetic products was investigated in detail. A variety of the resident and transient microbial flora of the skin, oral cavity, and intestine was applied to an investigation of the antimicrobial activity of C16:1 $\Delta 6$. C16:1 $\Delta 6$ showed selective antibacterial activity against human microbial pathogens such as Clostridium perfringens (one of the most common causes of food poisoning) and Streptococcus mutans (one of the tooth-decaying bacteria), as well as Staphylococcus aureus. C16:1 $\Delta 6$ seemed to possess a "species-selective" antibacterial activity against bacteria belonging to the genera Staphylococcus and Clostridium. We performed a preliminary assessment of the application of C16:1 $\Delta 6$ as an antimicrobial component in a liquid lip gloss by performing preservation efficacy and home-use tests. We noted that the amount of $p$-hydroxybenzoate was needed in an oily cosmetic, especially liquid lip gloss, could be reduced by replacing $p$-hydroxybenzoate with C16:1 $\Delta 6$. We suggest that C16:1 $\Delta 6$ may find application as a stable antimicrobial substance gentle enough for use in consumer products by its selective antimicrobial characteristics.
\end{abstract}

\section{Keywords}

Human Sebaceous Fatty Acid, Cis-6-Hexadecenoic Acid, Oily Cosmetics, Liquid Lip Gloss, Preservative Efficacy

\section{Introduction}

Cis-6-hexadecenoic acid (C16:1 $\Delta 6)$ (Figure 1) is the most abundant fatty acid in 


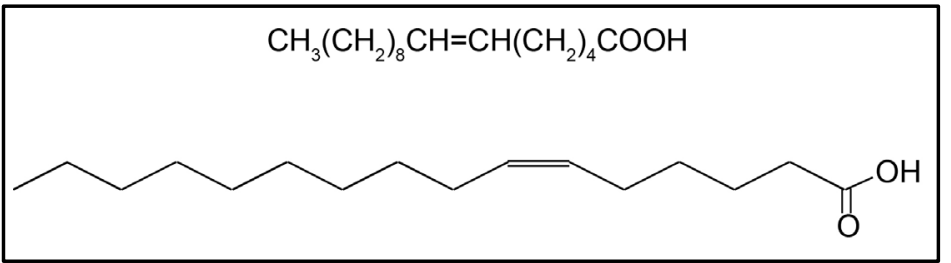

Figure 1. Chemical structure of cis-6-hexadecenoic acid (C16:1 $\Delta 6)$.

human sebum and is ubiquitous in human skin and hair [1].

This fatty acid is also called sapienic acid because the presence of C16:1 $\Delta 6$ is restricted to humans among hair-bearing animals [2]. This fatty acid is formed enzymatically from palmitic acid by $\Delta-6$ desaturase, which is expressed as the major fatty acid desaturase in human sebaceous glands [3]. It seems likely that sebum plays a specific role in the innate defense mechanisms of healthy human skin against foreign microbes [4] and that sebum-derived unsaturated fatty acids (such as $\mathrm{C} 16: 1 \Delta 6$ ) contribute to the antimicrobial activity exhibited by the skin [5] [6] [7] [8] [9]. C16:1 $\Delta 6$ has not been found in ordinary vegetable and animal oils with the exception of the seed oil derived from Thunbergia alata, commonly called the Black-eyed Susan vine. Indeed, methods for the production of C16: $1 \Delta 6$ historically have been restricted to extraction from the seed oil and a few chemical syntheses [10] [11].

As reported elsewhere, we have developed methods for the convenient production of C16:1 $\Delta 6$ by means of industrial processes [12] [13]. A mutant of the alkane-assimilating Rhodococcus sp. KSM-B-3 was shown to introduce a cisdouble bond at the 6th position of isopropyl hexadecanoate as a new activity; the resulting isopropyl hexadecenoate (IP-C16:1 $\Delta 6$ ) was readily hydrolyzed to C16: $1 \Delta 6$.

In a study of the relationship between sebum composition and skin disease, we found that there was a significant decrease in free $C 16: 1 \Delta 6$ content in the skin of patients with atopic dermatitis (AD) compared with that of healthy controls [14]. The lower level of free C16:1 $\Delta 6$ seemed to correlate with the higher numbers of Staphylococcus aureus in the skin of AD patients. A pilot clinical test using topically applied C16:1 $\Delta 6$ yielded reduced $S$. aureus numbers in $75 \%$ of the $\mathrm{AD}$ patients tested. Therefore, we hypothesize that free $\mathrm{C} 16: 1 \Delta 6$ may be involved in the normal human defense against $S$. aureus.

In the present paper, we characterize the antimicrobial properties of C16:1 $\Delta 6$ against the resident and transient microbial flora of humans. Additionally, we suggest the feasibility of using C16:1 $\Delta 6$ with $p$-hydroxybenzoate as an antimicrobial substance in cosmetic products. Though $p$-hydroxybenzoate is widely used as a chemically synthesized preservative in a variety of cosmetic and food products, the suggested combination with this antimicrobial substance would decrease the use of $p$-hydroxybenzoate, which may be advantageous from the point of view of safety and ecology. Thus, the use of C16:1 $\Delta 6$, an innate component of the human metabolome, may find application in the suppression of microorganisms harmful to human health while reducing the need for synthesis 
and dissemination of synthetic preservatives.

\section{Materials and Methods}

\subsection{Preparation of $\mathrm{C} 16: 1 \Delta 6$}

This study used Rhodococcus sp. strain KSM-T64 (FERM P-18182 in NITE), a mutant impaired for esterase production that was derived from the alkane-assimilating Rhodococcus sp. strain KSM-B-3 (FERM BP-1531 in NITE) [13]. The KSM-B-3 parent strain was itself isolated from a soil sample, as previously described [13]. Cultures of Rhodococcus sp. strain KSM-T64 were grown in medium, which contained $2.0 \%$ monosodium glutamate, $2 \mathrm{mM} \mathrm{MgSO}_{4} \cdot 7 \mathrm{H}_{2} \mathrm{O}, 2 \mu \mathrm{M}$ $\mathrm{MnSO}_{4} \cdot 6 \mathrm{H}_{2} \mathrm{O}, 60 \mu \mathrm{M} \mathrm{FeSO}_{4} \cdot 7 \mathrm{H}_{2} \mathrm{O}, 5 \mu \mathrm{M} \mathrm{CuSO}_{4} \cdot 5 \mathrm{H}_{2} \mathrm{O}, 0.8 \%$ yeast extract (Asahi Beer, Tokyo, Japan), $0.35 \mathrm{M}$ potassium phosphate buffer ( $\mathrm{pH} 7.3$ ), and $22 \%$ isopropyl hexadecanoate (Tokyo Chemical Industry, Tokyo, Japan), permitting bioconversion to IP-C16:1 $\Delta 6$ with shaking at $26^{\circ} \mathrm{C}$. Following isolation from the spent culture medium, the resulting IP-C16:1 $\Delta 6$ was hydrolyzed under alkaline condition $\left(80^{\circ} \mathrm{C}, 3 \mathrm{~h}\right)$ to generate $\mathrm{C} 16: 1 \Delta 6$. This product then was purified by thin-film distillation $\left(140^{\circ} \mathrm{C}-160^{\circ} \mathrm{C}, 3 \mathrm{~Pa}\right)$. The $\mathrm{C} 16: 1 \Delta 6$ used in the present study was approximately $95 \%$ pure.

\subsection{Microorganisms}

Microorganisms mentioned below were used for the investigation of the antimicrobial efficacy of C16:1 $\Delta 6$. Representatives of the bacteria typically found on the human skin were as follows: Staphylococcus epidermidis JCM 2414, Staphylococcus saprophyticus JCM 2427, S. aureus ATCC 12600, Micrococcus luteus JCM 1464, Micrococcus varians IFO 15358, Corynebacterium xerosis JCM 1971, and Propionibacterium acnes ATCC 6919. Representatives of the bacteria found in the human oral cavity were as follows: Streptococcus mutans JCM 5705, Streptococcus salivarius JCM 5707, Streptococcus sanguis JCM 5708, Actinomyces viscosus ATCC 15987, and Actinomyces naeslundii ATCC 12104. Representatives of bacteria found in the human intestine were as follows: Clostridium perfringens JCM 1290, Clostridium butyricum JCM 1391, Escherichia coli ATCC 11775, and Enterobacter cloacae JCM 1232. Representatives of spore-forming bacteria broadly detected in the environment were as follows: Bacillus subtilis IFO 13719 and Bacillus cereus JCM 2152. Representatives of bacteria generally found in aqueous environments were as follows: Pseudomonas aeruginosa IFO 12689, Pseudomonas putida ATCC 14164, Pseudomonas cepacia JCM 5964, and Pseudomonas fluorescens JCM 5963. Representatives of the yeasts were as follows: Candida albicans JCM 1542, Saccharomyces cerevisiae JCM 7255, and Cryptococcus laurentii IFO 0609.

In addition, three unidentified environmental strains (isolated during homeuse testing of liquid lip gloss) were employed as the test strains for the preservative efficacy testing. These strains consisted of two Gram-positive coccal bacteria (Staphylococcus sp. and Streptococcus sp. designated 030411-1-1 and 0304111-2) as well as a Gram-positive rod-shaped bacterium (Bacillus sp. designated 
030411-1-3).

\subsection{Media}

SCD and SCDLP agar media purchased from Nihon Pharmaceutical (Tokyo, Japan) were used for bacterial cultivation, except for $P$. acnes, which used GAM Agar Modified "Nissui" purchased from Nissui Pharmaceutical (Tokyo, Japan). GP and GPLP agar medium purchased from Nihon Pharmaceutical were used for culturing yeasts.

\subsection{Chemicals}

Chemicals for cultivation of Rhodococcus sp. strain KSM-T64 were of analytical grade and obtained from Wako Pure Chemical (Osaka, Japan) unless otherwise noted. Oleic acid (C18:1 $\Delta 9)$ used for the study of antimicrobial activity was obtained as an analytical grade compound from Sigma (St. Louis, MO, USA), and other materials formulated in cosmetic formulations were of cosmetic grade.

\subsection{Antimicrobial Activity of C16:1 $1 \Delta 6$}

Freshly growing $\left(2-3\right.$ days on $\mathrm{SCD}$ agar at $\left.30^{\circ} \mathrm{C}\right)$ microbes were suspended at $10^{6} \mathrm{cfu} / \mathrm{mL}$ in $50 \mathrm{mM}$ phosphate buffer $(\mathrm{pH} 7.0)$, and suspensions were mixed well. C16:1 $\Delta 6$ was prepared as a $10 \%(\mathrm{v} / \mathrm{v})$ solution in ethanol and added to each bacterial suspension at a final concentration of 25 or $50 \mathrm{mg} / \mathrm{L}$. After a 1-h incubation at $30^{\circ} \mathrm{C}$, a portion of the suspension was sampled. After inactivation of antimicrobial activity of C16:1 $\Delta 6$ in the sample by Lecithin and Polysorbate 80 (LP) diluent "Daigo" (Nihon Pharmaceutical), the sample was diluted in LP diluent and aliquots of the inactivated sample were spread onto an appropriate agar medium supplemented with LP diluent "Daigo". After incubation at $30^{\circ} \mathrm{C}$ for 2 - 3 days (except for $P$. acnes, which was incubated at $37^{\circ} \mathrm{C}$ for $3-5$ days), the number of colonies on agar plates were counted, used to calculate cell densities, and then compared to that of control without C16:1 $\Delta 6$. Cultivation of anaerobic microbes was performed using the "Anaero Pack" anaerobic cultivation system (Mitsubishi Gas Chemical, Tokyo, Japan).

\subsection{Comparison of Antimicrobial Activity of C16:1 16 and Oleic Acid $(18: 1, \Delta 9)$}

Freshly growing $\left(2-3\right.$ days on SCD agar at $\left.30^{\circ} \mathrm{C}\right)$ microbes were suspended at $10^{7} \mathrm{cfu} / \mathrm{mL}$ in saline and used as inocula at $1 \%(\mathrm{v} / \mathrm{v})$ in individual aliquots of 50 $\mathrm{mM}$ phosphate buffer $(\mathrm{pH} 6.0)$. C16:1 $\Delta 6$ and oleic acid $(18: 1, \Delta 9)$ were prepared as a $10 \%(\mathrm{v} / \mathrm{v})$ solution in ethanol and added to each bacterial suspension at a final concentration of 10,25 , or $50 \mathrm{mg} / \mathrm{L}$. Following inoculation, the sample was incubated at $30^{\circ} \mathrm{C}$ and sampled at 1,4 , and 24 hours in order to confirm the antimicrobial efficacy of each reagent in a short period. Specimens from respective time points were diluted by LP diluent, plated, incubated, and counted as above. An acceptable range of deviation of $0.5 \mathrm{log}$, which was mentioned in ISO11930, was applied to this preservative efficacy test. 


\subsection{Preservation Efficacy and Home-Use Tests}

Cosmetic products such as a liquid lip gloss typically are supplied in a container with a built-in brush, and used in a manner in which the application brush is expected to back-contaminate the container with skin-derived materials and microorganisms. To inhibit the growth of microorganisms, appropriate counter-plans must be constructed. To evaluate the antimicrobial efficacy of C16:1 $\Delta 6$ under these application conditions, model liquid lip glosses with different preservative components were assayed using a preservative efficacy test (a general laboratory testing method [15]) and a home-use test.

Preservative efficacy test: Model liquid lip gloss was prepared as a combination of $60 \%$ heavy fluid isoparaffin, $8 \%$ ceresin, $0.5 \%$ stearyl glycyrrhetinate, $2 \%$ red No. 202, 2\% oxidized titanium, $7 \%$ mica coated with oxidized titanium, $0.5 \%$ hyaluronic acid, and squalene (balance); this is the base control formulation 1. Aliquots with different preservative system were formulated to include either the combination: formulation 3 with ethyl paraben and propyl paraben (preservatives commonly used in personal care and cosmetic formulations) at $0.05 \%(\mathrm{w} /$ w) each or formulation 2 with C16:1 16 at $0.1 \%(w / w)$ (Table 1$)$.

Bacterial strains consisted of Gram-positive environmental isolates 030411-11, 030411-1-2, or 030411-1-3, which were derived from home-use testing of liquid lip gloss. Freshly growing bacteria (cultivated on SCD agar plates at $30^{\circ} \mathrm{C}$ for 2 - 3 days) were suspended at $10^{8} \mathrm{cfu} / \mathrm{mL}$ in saline and used as inocula at $1 \%$ $(\mathrm{v} / \mathrm{w})$ in individual aliquots of liquid lip gloss. Following inoculation, the lip gloss was incubated at $30^{\circ} \mathrm{C}$ and sampled at $1,2,3$, and 5 days in order to confirm the efficacy of preservative system in a short period. Specimens from respective time points were diluted by LP diluent, plated, incubated, and counted as above. An acceptable range of deviation of $0.5 \mathrm{log}$, which was mentioned in ISO11930, was applied to this preservative efficacy test.

Home-use test: Liquid lip gloss was formulated as above but using (as preservatives) one of the three following preservative systems (Table 1): formulation 5 with ethyl and propyl parabens at $0.1 \%(\mathrm{w} / \mathrm{w})$ each; formulation 6 with $0.1 \%$ $(\mathrm{w} / \mathrm{w})$ ethyl and propyl parabens $+0.5 \%(\mathrm{w} / \mathrm{w})$ oleic acid (C18:1 $\Delta 9$; an unsaturated fatty acid commonly used for cosmetics); or formulation 4 with $0.5 \%$ $(\mathrm{w} / \mathrm{w}) \mathrm{C} 16: 1 \Delta 6+0.1 \%(\mathrm{w} / \mathrm{w})$ propyl paraben. Female panel members $(\mathrm{n}=20)$

Table 1. Preservative system of model liquid-type lip gloss for preservative efficacy and home-use tests.

\begin{tabular}{ccccccc}
\hline & \multicolumn{3}{c}{$\begin{array}{c}\text { Preservative system } \\
\text { for preservative efficacy test }\end{array}$} & \multicolumn{3}{c}{$\begin{array}{c}\text { Preservative system } \\
\text { for home-use test }\end{array}$} \\
\hline $\begin{array}{c}\text { Ingredient } \\
(\%(\mathrm{w} / \mathrm{v}))\end{array}$ & 1 & 2 & 3 & 4 & 5 & 6 \\
\hline $\mathrm{C} 16: 1 \Delta 6$ & - & 0.1 & - & 0.5 & - & - \\
Oleic acid & - & - & - & - & - & 0.5 \\
Ethylparaben & - & - & 0.05 & - & 0.1 & 0.1 \\
Propylparaben & - & - & 0.05 & 0.1 & 0.1 & 0.1 \\
\hline
\end{tabular}


applied a given formulation twice per day for 2 weeks. The same group then applied (in the same manner) a separate formulation on each of two subsequent two-week intervals. Written informed consent was obtained from all subjects in accordance with the Declaration of Helsinki following an explanation of the experimental protocols. The study and protocols were approved by our institutional ethics committee. After each 2-week interval, the numbers of panel members who reported incompatibility (e.g., increased irritation) were recorded, and malodor of the returned samples was evaluated by sensual inspection. Water evaporation from one lip of each panel member was determined (both before and after application of $0.05 \mathrm{~g}$ of each formulation) using a humidity sensor with a quartz crystal (Skicon-200, IBS Co. Ltd, Shizuoka, Japan) at $30^{\circ} \mathrm{C}$ with $30 \%$ $\mathrm{RH}$. And the efficacy of suppression of water evaporation from the lip was calculated by using below Equation (1).

The efficacy of suppression of water evaporation (\%)

$=(1-($ water evaporation volume before application/water evaporation volume after application $)) \times 100$

Significant differences between experimental values for the number of malodorous samples were determined using chi-square test for goodness of fit, and p-values $<0.05$ were considered as the level of significance.

\section{Results and Discussion}

\subsection{Antimicrobial Properties of C16:1 16}

Antimicrobial activity of C16:1 $\Delta 6$ against 25 kinds of microorganisms corresponding to the resident and transient human flora was evaluated in detail at two different concentrations; 25 or $50 \mathrm{mg} / \mathrm{L}$. It was reported that C16:1 $1 \Delta 6$ had a synergistic effect with high concentration of ethanol [16], so additive amount of ethanol solution including $\mathrm{C} 16: 1 \Delta 6$ was limited at very low in order to avoid the synergistic effect with ethanol and the antimicrobial activity of ethanol itself. As a result, C16:1 $\Delta 6$ was not effective against Gram-negative microbes, but did demonstrate activity against Gram-positive microbes as previously reported [8] [9] [14]. This selective antimicrobial efficacy was found at two different concentrations, so the result at $50 \mathrm{mg} / \mathrm{L}$ was shown in Table 2.

The compound exhibited a very unique "species-selective" antimicrobial activity (that is, antimicrobial effectiveness that differed among species in the same genus), as shown by comparison between $C$. perfringens and $C$. butyricum and between $S$. epidermidis and $S$. aureus, "species-selectivity" for $S$. epidermidis and $S$. aureus was already found by Takigawa et al. [14], so the selectivity for Clostridium species was newly discovered in this experiment. In the case of the Clostridium species, the results were consistent with other information about favored intestinal microflora and intestinal health: C16:1 $\Delta 6$ was effective against $C$. perfringens which is known as an unwelcome resident in the intestine, but the compound was less effective against $C$. butyricum, which has been used in probiotics [17]. From this result, we could find a novel advantage of this unique fatty acid. 
Table 2. Antimicrobial efficacy of $\mathrm{C} 16: 1 \Delta 6$ against various microorganisms.

\begin{tabular}{|c|c|c|c|}
\hline & No. & Test strain & $\begin{array}{l}\text { Ratio } \\
(\%)^{\mathrm{a}}\end{array}$ \\
\hline \multirow{7}{*}{ Skin microbe } & 1 & Staphylococcus epidermidis JCM 2414 & 16.7 \\
\hline & 2 & Staphylococcus saprophyticus JCM 2427 & 18.9 \\
\hline & 3 & Staphylococcus aureus ATCC 12600 & 1.90 \\
\hline & 4 & Micrococcus luteus JCM 1464 & $<0.100$ \\
\hline & 5 & Micrococcus varians IFO 15358 & $<0.100$ \\
\hline & 6 & Corynebacterium xerosis JCM 1971 & $<0.100$ \\
\hline & 7 & Propionibacterium acnes ATCC 6919 & $<0.100$ \\
\hline \multirow{5}{*}{ Oral microbe } & 8 & Streptococcus mutans JCM 5705 & $<0.100$ \\
\hline & 9 & Streptococcus salivarius JCM 5707 & $<0.100$ \\
\hline & 10 & Streptococcus sanguis JCM 5708 & $<0.100$ \\
\hline & 11 & Actinomyces viscosus ATCC 15987 & $<0.100$ \\
\hline & 12 & Actinomyces naesulundii ATCC 12104 & $<0.100$ \\
\hline \multirow{4}{*}{ Intestinal microbe } & 13 & Clostridium perfringens JCM 1290 & $<0.100$ \\
\hline & 14 & Clostridium butyricum JCM 1391 & 42.2 \\
\hline & 15 & Escherichia coli ATCC 11775 & 16.4 \\
\hline & 16 & Enterobacter cloacae JCM 1232 & 26.7 \\
\hline \multirow{2}{*}{$\begin{array}{l}\text { Spore-forming } \\
\text { microbe }\end{array}$} & 17 & Bacillus subtilis IFO 13719 & $<0.100$ \\
\hline & 18 & Bacillus cereus JCM 2152 & $<0.100$ \\
\hline \multirow{4}{*}{$\begin{array}{l}\text { Environmental } \\
\text { microbe }\end{array}$} & 19 & Pseudomonas aeruginosa IFO 12689 & 32.5 \\
\hline & 20 & Pseudomonas putida ATCC 14164 & 37.3 \\
\hline & 21 & Pseudomonas cepacia JCM 5964 & 4.10 \\
\hline & 22 & Pseudomonas fluorescens JCM 5963 & 10.0 \\
\hline \multirow{3}{*}{ Yeast } & 23 & Candida albicans JCM 1542 & 2.20 \\
\hline & 24 & Saccharomyces cerevisiae JCM 7255 & $<0.100$ \\
\hline & 25 & Cryptococcus laurentii IFO 0609 & $<0.100$ \\
\hline
\end{tabular}

${ }^{\text {a }}$ Value shows ratio of surviving microorganisms. Reaction was performed at $30^{\circ} \mathrm{C}$ for $1 \mathrm{~h}$ at the final concentration of $50 \mathrm{mg} / \mathrm{L}$ ( $\mathrm{pH} 7.0$ ); initial inoculum was $10^{6} \mathrm{cfu} / \mathrm{mL}$.

Species-selective antimicrobial activity is known in the field of antifungal derivatives against Tricophyton species. At low concentration, miconazole nitrate, a popular imidazole antifungal derivative, shows good antimicrobial efficacy against Trichophyton mentagrophytes but not against Trichophyton rubrum [18]. This kind of selectivity is thought to be caused by the difference in the unsaturation ratio of the organisms' lipids and of affinity for cell membrane lipids such as sterols [19]. Miconazole has been proposed to target the plasma membrane, causing membrane disruption by inhibiting the synthesis of sterol, with subsequent induction of metabolism imbalance [19]. Our mechanistic analysis was based on analogy to the assumed antimicrobial mechanism of antifungal agents (data not shown).

We also found that $\mathrm{C} 16: 1 \Delta 6$ had high efficacy against $P$. acnes, which is 
known to be a factor in acne, and against $C$. albicans, which is known to be a main cause of candidiasis. Given that $\mathrm{C} 16: 1 \Delta 6$ application to the skin of $\mathrm{AD}$ patients has been shown to inhibit colonization by $S$. aureus [14], we propose to confirm the effectiveness of topical application of C16:1 $\Delta 6$ against these transient microbes via in vivo testing in the near future.

Considering our results in the context of the literature, we infer that C16:1 $\Delta 6$ maintains normal skin conditions both by promoting water retention and by playing a defensive role via mild antimicrobial efficacy. This proposed mechanism would be similar to that of sphingosine and related ceramide lipid derivatives, which are known to contribute to the normalization of skin; those compounds show antimicrobial efficacy against various microbes on the skin and in the oral cavity [20]. Indeed, skin levels of sphingosine have been shown to be decreased in patients with $\mathrm{AD}$ compared to healthy people; such decreases induce colonization of the skin by microbes such as $S$. aureus [21]. The defense mechanism by a human sebaceous lipid is very interesting in the context of homeostasis on the skin. We hypothesize that C16:1 $\Delta 6$ will be superior to other unsaturated fatty acids, given that $\mathrm{C} 16: 1 \Delta 6$ is ubiquitous on human skin and hair while also showing species-selective antimicrobial properties.

\subsection{Comparison of Antimicrobial Activity of C16:1 146 and Oleic Acid Frequently Used in Cosmetic Products}

We compared the antimicrobial activity of C16:1 16 and oleic acid, which was frequently used in cosmetic products, against $S$. aureus and $S$. epidermidis at 10, 25 , and $50 \mathrm{mg} / \mathrm{L}$ respectively. As a result of this experiment, C16:1 $\Delta 6$ showed higher antimicrobial efficacy against $S$. aureus than oleic acid at 1 and 4 hours with significant differences. On the other hand, both unsaturated fatty acid showed less antimicrobial efficacy against $S$. epidermidis compared to $S$. aureus. This "species selective antimicrobial efficacy" was confirmed at any concentrations, so the result at $50 \mathrm{mg} / \mathrm{L}$ was shown in Figure 2.

From this result, we found that human innate C16:1 $\Delta 6$ had significantly higher selective antimicrobial activity than widely-used oleic acid for the first time.

There are some reports for antimicrobial activity of oleic acid. High antimicrobial efficacy against oral microorganisms [22] and methicillin-resistant Staphylococcus aureus (MRSA) [23] was reported, and it was suggested that the cause of this efficacy was derived from attack and disruption of cell wall. In addition, there are several reports about the antimicrobial activity of unsaturated fatty acids against $S$. aureus. In these reports, the unsaturated fatty acids interfered with energy production within the bacterial cells by disrupting the cell membrane and adaptation of $S$. aureus to these acids correlated with reduced hydrophobicity of the cell surface [24]. Another report indicated that the antimicrobial activity of 16:1 $\Delta 9$ against $S$. aureus was caused by depolarization of the cell membrane and the release of low molecular proteins [25]. That report suggested that changes in the teichoic acid content of the cell wall provided protection against the antibacterial activity of a fatty acid. We compared the fatty acid 


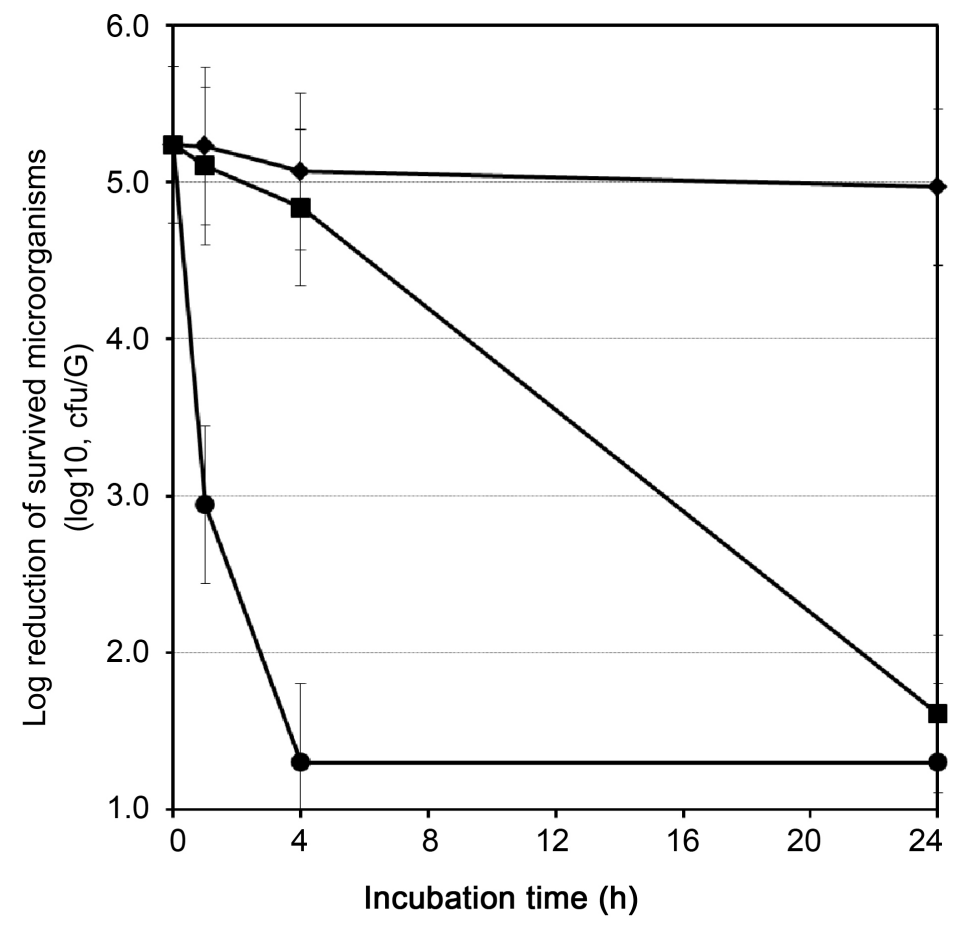

(a)

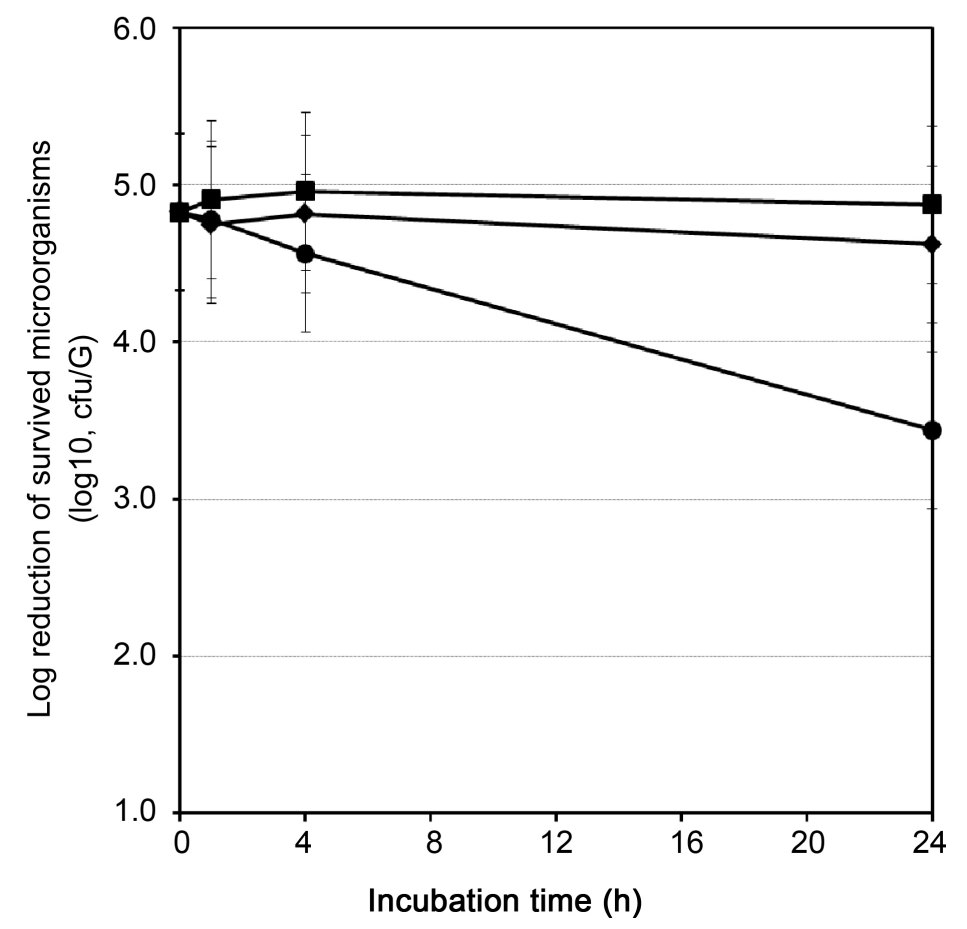

(b)

Figure 2. Antimicrobial efficacy of C16:1 $\Delta 6$ and oleic acid (18:1, $\Delta 9)$ against $S$. aureus (a) and $S$. epidermidis (b). $\diamond$ Control (without fatty acid); $\bullet, 50 \mathrm{mg} / \mathrm{L} \mathrm{C16:1} \Delta$; •, $50 \mathrm{mg} / \mathrm{L}$ oleic acid. Error bar shows an acceptable range of deviation of $0.5 \mathrm{log}$ which was mentioned in ISO 11930.

compositions of $S$. aureus and $S$. epidermidis. The linear-chain fatty acid content of $S$. aureus was higher in than that of $S$. epidermidis. In contrast, branched- 
chain fatty acids content was lower in $S$. aureus compared to $S$. epidermidis, with values for iso-forms and for anteiso-forms (data not shown). Fatty acids are known to be the main components of the cell membrane, and have been shown to change in response to antimicrobial stressors such as antibiotics. We inferred that the high ratio of anteiso-fatty acids in $S$. epidermidis contributes to decreased susceptibility to C16:1 $1 \Delta 6$ compared to that of $S$. aureus. In addition, it was reported that the decrease of branched-chain membrane fatty acid content increased susceptibility to a variety of stresses by producing more-rigid membrane in S. aureus [26]. The difference in the fatty acid composition of Staphylococcus species may lead to the differences in susceptibility to C16:1 $\Delta 6$. Even though most antimicrobial substances have multiple sites of action and cells rendered non-viable by many means will express such phenomena, the present work may suggest that the antibacterial activity of C16:1 $\Delta 6$ was mediated by effects on the cell membrane, and that the activity of C16:1 $\Delta 6$ correlated with the level of branched-chain fatty acids in the cell membrane of Staphylococcus species.

\subsection{Incorporation of $\mathrm{C} 16: 1 \Delta 6$ into a Cosmetic Applied to the Mucous Membrane}

In general, non-aqueous formulations do not contain water and show low water activity (Aw: $<0.75$ ); incorporated microorganisms cannot grow in such formulations, and cell counts decrease gradually even in formulations that lack preservatives [27]. But sometimes it is thought that quality deterioration of products will be caused by microbial growth and generation of malodor under high humid condition with incorporated lip component and saliva. In that case, contamination by Gram-negative microbes is typically rarer than that by Gram-positive microbes, given that Gram-negative bacteria exhibit poorer survival in low water activity conditions. So it may be possible that liquid lip gloss is susceptible to contamination by Gram-positive microbes; we therefore employed environmental isolates of Gram-positive microbes in an evaluation of the preservative efficacy of model liquid lip gloss formulations that included C16:1 $\Delta 6$.

In a preservation efficacy test using any of three Gram-positive bacterial isolates, it was found that the formulation 2 including $\mathrm{C} 16: 1 \Delta 6$ at the concentration of $0.1 \%(\mathrm{w} / \mathrm{w})$ showed a rapid decrease in cell number compared to the control formulation 1 without preservatives (Figure 3).

A similar preservative efficacy was observed in a formulation 3 that incorporated ethyl and propyl parabens at concentrations of $0.05 \%(w / w)$ each (Figure 3). In this experiment, remarkable difference of efficacy between formulation 1 and formulation 2, between formulation 1 and formulation 3 , or between formulation 2 and formulation 3 was not achieved considering an acceptable range of deviation of $0.5 \log$ which was mentioned in ISO11930 [15], however C16:1 $\Delta 6$ demonstrated a stable antimicrobial efficacy in a cosmetic formulation by this reproducible in vitro tests. In addition, the formulations used in this study showed rapid decrease for some standard Gram-negative test strains such as $E$. coli and $P$. aeruginosa by using a typical preservative efficacy test [15] and were 


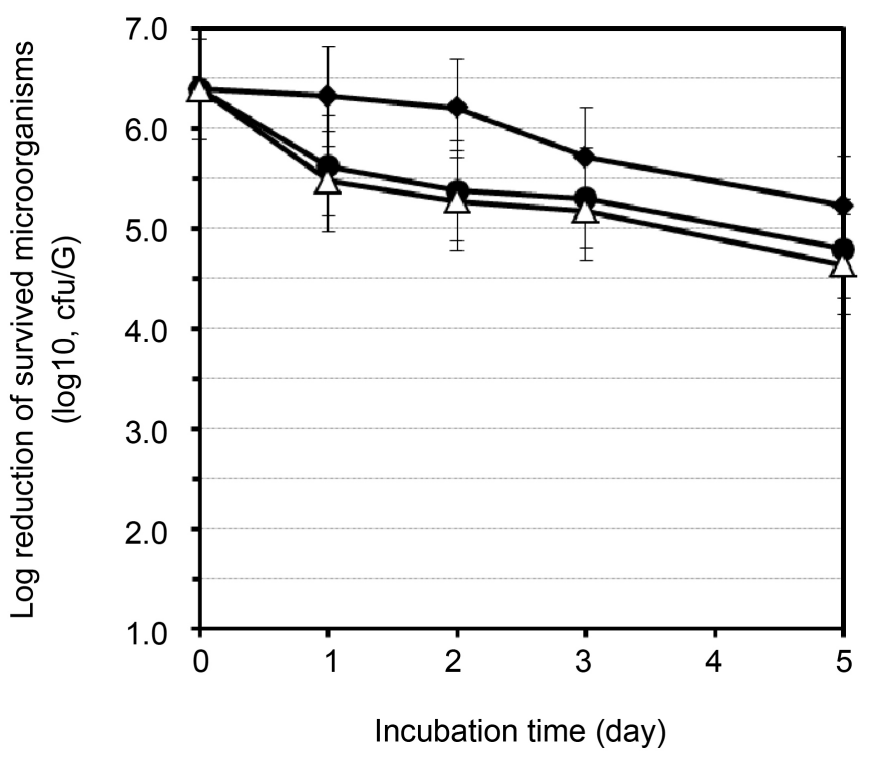

Figure 3. Preservative efficacy of liquid-type lip gloss products formulated with C16:1 $\Delta 6$. Three distinct Gram-positive environmental isolates (strains 030411-1-1, 030411-1-2, and 030411-1-3) were tested. $\bullet$ Control formulation 1 without preservatives; $\bullet$, formulation 2 with $0.1 \%(\mathrm{w} / \mathrm{w}) \mathrm{C} 16: 1 \Delta 6 ; \Delta$, formulation 3 with $0.05 \%$ $(\mathrm{w} / \mathrm{w})$ ethyl paraben $+0.05 \%(\mathrm{w} / \mathrm{w})$ propyl paraben. Error bar shows an acceptable range of deviation of $0.5 \mathrm{log}$ which was mentioned in ISO 11930.

confirmed to have an adequate preservative efficacy for these microorganisms (data not shown).

Home-use tests were used to compare the use of three distinct preservative systems: formulation 4 was $0.5 \%(\mathrm{w} / \mathrm{w}) \mathrm{C} 16: 1 \Delta 6+0.1 \%(\mathrm{w} / \mathrm{w})$ propyl paraben; formulation 5 was the combination of ethyl and propyl parabens at $0.1 \%(\mathrm{w} / \mathrm{w})$ each; and formulation 6 was $0.1 \%(\mathrm{w} / \mathrm{w})$ ethyl and propyl parabens $+0.5 \%(\mathrm{w} / \mathrm{w})$ oleic acid. None of the C16:1 $\Delta 6$-containing samples displayed a malodor after two weeks of home use; in contrast, the other two formulations (parabens only and parabens + oleic acid) exhibited malodor in 7 of 20 and 4 of 20 samples respectively. By using chi-square test for goodness of fit, it was found that there was a significant difference with $\mathrm{p}$-value $<0.05$ for the generation of malodor in these formulations. In addition, no panel members reported an irritation during two weeks of twice-daily application (Table 3).

The formulation 4 with C16:1 $\Delta 6$ showed a higher antimicrobial efficacy in the home-use test compared to the formulation with oleic acid. Based on these results, human sebaceous fatty acid C16:1 $\Delta 6$ functioned effectively in a real-use situation while providing mild preservative efficacy for users. We also found that the preservative system with $\mathrm{C} 16: 1 \Delta 6$ showed good suppression of water evaporation from the lip compared to the system using paraben esters only or paraben esters + oleic acid; formulation 4 showed 52\%, formulation 5 showed $45 \%$, and formulation 6 showed $38 \%$ as an average ratio of suppression of water evaporation from the lip. We envision a bright future for the development of products 
Table 3. Result of home-use testing with 3 model formulations.

\begin{tabular}{cccc}
\hline & \multicolumn{3}{c}{ Formulation for home-use test } \\
\cline { 2 - 4 } & 4 & 5 & 6 \\
\hline $\begin{array}{c}\text { Number of malodorous samples } \\
\text { (Ratio of malodorous samples) }\end{array}$ & $\begin{array}{c}0 \text { of } 20 \\
(0 \%)\end{array}$ & $\begin{array}{c}7 \text { of } 20 \\
(35 \%)\end{array}$ & $\begin{array}{c}4 \text { of } 20 \\
(20 \%)\end{array}$ \\
Number of people felt irritation & 0 of 20 & 0 of 20 & 0 of 20 \\
\hline
\end{tabular}

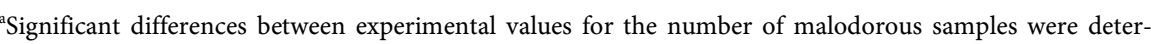
mined using chi-square test for goodness of fit, with $\mathrm{p}<0.05$ considered significance. In this experiment, chi-square test for goodness of fit was 6.73 and the p-value was 0.03 .

incorporating this unique unsaturated fatty acid as an antimicrobial agent.

Cosmetics for lips, especially liquid lip gloss, are used for long periods; as a result, saliva, components from lips, and microbes on skin and in the oral cavity might be introduced into a formulation. In that situation, contaminants might negatively influence product quality (e.g., create malodor), depending on the product type, package design, miscibility with water, product usage, etc. To repress a malodor because of growth of microorganisms, it is very effective to add preservatives into a formulation. On the other hand, the lips represent very sensitive mucous membranes; incorporation of preservatives could result in increased irritation and/or damage to the lips. We aimed to improve the preservative efficacy of a formulation in oily liquid lip cosmetics by including C16:1 $\Delta 6$, a compound that already is present on human skin and displays appealing antimicrobial efficacy. There are many Gram-positive microbes that are resident flora on the skin (e.g., Staphylococcus species) and in the oral cavity (e.g., Streptococcus species). The addition of C16:1 $\Delta 6$ to cosmetics such as lip gloss is expected to be a very useful approach, and we confirmed the actual effectiveness of this fatty acid. GC analyses show that $\mathrm{C} 16: 1 \Delta 6$ is stable in oily liquid cosmetics (e.g., liquid lip gloss), which are composed of various oily raw materials, and that C16:1 $\Delta 6$ is stable when formulated as a simple solution that incorporates an anti-oxidizing agent such as dibutylhydroxytoluene (data not shown). Our clarification of the antimicrobial properties of C16:1 $\Delta 6$ should facilitate the application of this molecule as a component of consumer products.

\section{Conclusion}

C16:1 $\Delta 6$ had species-selective antimicrobial efficacy against not only staphylococcal but also clostridial species. Notably, C16:1 $\Delta 6$ showed an appealing profile of antimicrobial activity against objectionable microbes that have potential pathogenicity in humans, including those found on skin and in the oral cavity. By performing preservation efficacy and home-use tests, formulations incorporating C16:1 $\Delta 6$ inhibited growth of spiked Gram-positive bacteria contaminants, and did not exhibit malodor or irritation during two weeks of human use. Based on these results, we suggest that this molecule may find application as a stable antimicrobial substance gentle enough for use in consumer products. 


\section{Acknowledgements}

We thank Dr. K. Yamamoto of Osaka Prefecture University (Osaka, Japan) for the investigation of an antimicrobial mechanism by analyzing bacterial fatty acid composition.

\section{Conflict of Interest}

The authors declare that they have no conflict of interest.

\section{References}

[1] Downing, D.T. and Strauss, J.S. (1974) Synthesis and Composition of Surface Lipids of Human Skin. Journal of Investigative Dermatology, 62, 228-244. https://doi.org/10.1111/1523-1747.ep12676793

[2] Carol, L.F., Derek, R.B., and Kim, A.B. (2014) The Roles of Cutaneous Lipids in Host Defense. Biochimica et Biophysica Acta, 1841, 319-322.

[3] Lan, G., Joel, S.G., Charleen, H., Kurt, S., and Stephen, M.P. (2003) Identification of the $\Delta-6$ Desaturase of Human Sebaceous Glands: Expression and Enzyme Activity. Journal of Investigative Dermatology, 120, 707-714. https://doi.org/10.1046/j.1523-1747.2003.12123.x

[4] John, J.W., David, D., and Philip, W.W. (1997) Identification of cis-Palmitoleic Acid as the Active Antimicrobial in Human Skin Sebum. Journal of Investigative Dermatology, 108, 677.

[5] Burtenshaw, J.M.L. (1942) The Mechanism of Self-Disinfection of Human Skin Its Appendages. Journal of Hygiene, 42, 184-210.

https://doi.org/10.1017/S0022172400035373

[6] Bergsson, G., Arnfinnsson, J., Steingrímsson, O., and Thormar, H. (2001) Killing of Gram-Positive Cocci by Fatty Acids and Monoglycerides. Acta Pathologica, Microbiologica, et Immunologica Scandinavica, 109, 670-678. https://doi.org/10.1034/j.1600-0463.2001.d01-131.x

[7] Chamberlain, N.R., Mehrtens, B.G., Xiong, Z., Kapral, F.A., Boardman, J.L., and Rearick, J.I. (1991) Correlation of Carotenoid Production, Decreased Membrane Fluidity, and Resistance to Oleic Acid Killing in Staphylococcus aureus 18Z. Infection and Immunity, 59, 4332-4337.

[8] Wille, J.J., and Kydonieus, A. (2003) Palmitoleic Acid Isomer (C16:1 $\Delta 6$ ) in Human Skin Sebum Is Effective against Gram-Positive Bacteria. Skin Pharmacology and Applied Skin Physiology, 16, 176-187. https://doi.org/10.1159/000069757

[9] Sumrell, G., Mod, R.R., Magne, F.C., and Novak, A.F. (1978) Antimicrobial Activity of Some Fatty Acid Derivatives. Journal of the American Oil Chemists' Society, 55, 395-397. https://doi.org/10.1007/BF02911899

[10] Daniela, K., Hansgeorg, E., and Jurgen, K. (2002) Process for the Preparation of cis-6-Hexadecenoic Acid. WO98/16104.

[11] Ernst, H., Klein, D. and Koppenhofer, J. (2002) Method for Producing cis-6-Hexadecenoic Acid. JP2002-241340.

[12] Koike, K., Takaiwa, M., Ara, K., Inoue, S., Kimura, Y. and Ito, S. (2000) Production of Isopropyl cis-6-Hexadecenoate by Regiospecific Desaturation of Isopropyl Palmitate by a Double Mutant of a Rhodococcus Strain. Bioscience, Biotechnology, and Biochemistry, 64, 399-404. https://doi.org/10.1271/bbb.64.399

[13] Koike, K., Ara, K., Adachi, S., Takigawa, H., Mori, H., Inoue, S., Kimura, Y. and Ito, 
S. (1999) Regiospecific Internal Desaturation of Aliphatic Compounds by a Mutant Rhodococcus Strain. Applied and Environmental Microbiology, 65, 5636-5638.

[14] Takigawa, H., Nakagawa, H., Kuzukawa, M., Mori, H. and Imokawa, G. (2005) Deficient Production of Hexadecenoic Acid in the Skin is Associated in Part with the Vulnerability of Atopic Dermatitis Patients to Colonization by Staphylococcus aureus. Dermatology, 211, 240-248. https://doi.org/10.1159/000087018

[15] ISO11930:2012 Cosmetics-Microbiology-Evaluation of the Antimicrobial Protection of a Cosmetic Product.

[16] Drake, D.R., Brogden, K.A., Dawson, D.V. and Wertz, P.W. (2008) Thematic Review Series: Skin Lipids. Antimicrobial Lipids at the Skin Surface. The Journal of Lipid Research, 49, 4-11. https://doi.org/10.1194/jlr.R700016-JLR200

[17] Kanauchi, O., Matsumoto, Y., Matsumura, M., Fukuoka, M. and Bamba, T. (2005) The Beneficial Effects of Microflora, Especially Obligate Anaerobes, and Their Products on the Colonic Environment in Inflammatory Bowel Disease. Current Pharmaceutical Design, 11, 1047-1053. https://doi.org/10.2174/1381612053381675

[18] Yamasaki, Y., Funai, K., Ueshima, M. and Shingu, H. (1979) Antimycotic Activity of Econazole Nitrate and Its Related Compounds. Nippon Saikingaku Zasshi, 34, 813-825. https://doi.org/10.3412/jsb.34.813

[19] Fromtling, R.A. (1988) Overview of Medically Important Antifungal Azole Derivatives. Clinical Microbiology Reviews, 1, 187-217. https://doi.org/10.1128/CMR.1.2.187

[20] Fischer, C.L., Drake, D.R., Dawson, D.V., Blanchette, D.R., Brogden, K.A. and Wertz, P.W. (2012) Antibacterial Activity of Sphingoid Bases and Fatty Acids Against Gram-Positive and Gram-Negative Bacteria. Antimicrobial Agents and Chemotherapy, 56, 1157-1161. https://doi.org/10.1128/AAC.05151-11

[21] Arikawa, J., Ishibashi, M., Kawashima, M., Takagi, Y., Ichikawa, Y. and Imokawa, G. (2002) Decreased Levels of Sphingosine, a Natural Antimicrobial Agent, May Be Associated with Vulnerability of the Stratum Corneum from Patients with Atopic Dermatitis to Colonization by Staphylococcus aureus. Journal of Investigative Dermatology, 119, 433-439. https://doi.org/10.1046/j.1523-1747.2002.01846.x

[22] Huang, C.B., George, B. and Ebersole, J.L. (2010) Antimicrobial Activity of n-6, n-7 and n-9 Fatty Acids and Their Esters for Oral Microorganisms. Archives of Oral Biology, 55, 555-560. https://doi.org/10.1016/j.archoralbio.2010.05.009

[23] Chen, C.H., Wang, Y., Nakatsuji, T., Liu, Y.T., Zouboulis, C., Gallo, R., Zhang, L., Hsieh, M.F. and Huang, C.M. (2011) An Iinnate Bactericidal Oleic Acid Effective against Skin Infection of Methicillin-Resistant Staphylococcus aureus. A Therapy Concordant with Evolutionary Medicine. Journal of Microbiology and Biotechnology, 21, 391-399.

[24] Kenny, J.G., Ward, D., Josefsson, E., Jonsson, I.M., Hinds, J., Rees, H.H., Lindsay, J.A., Tarkowski, A. and Horsburgh, M.J. (2009) The Staphylococcus aureus Response to Unsaturated Long Chain Free Fatty Acids: Survival Mechanisms and Virulence Implications. PLoS ONE, 4, e4344.

https://doi.org/10.1371/journal.pone.0004344

[25] Parsons, J.B., Yao, J., Frank, M.W., Jackson, P. and Rock, C.O. (2012) Membrane Disruption by Antimicrobial Fatty Acids Releases Low-Molecular-Weight Proteins from Staphylococcus aureus. Journal of Bacteriology, 194, 5294-5304. https://doi.org/10.1128/JB.00743-12

[26] Singh, V.K., Hattangady, D.S., Giotis, E.S., Singh, A.K., Chamberlain, N.R., Stuart, M.K. and Wilkinson, B.J. (2008) Insertional Inactivation of Branched-Chain AlphaKeto Acid Dehydrogenase in Staphylococcus aureus Leads to Decreased Branched- 
Chain Membrane Fatty Acid Content and Increased Susceptibility to Certain Stresses. Applied and Environmental Microbiology, 74, 5882-5890.

https://doi.org/10.1128/AEM.00882-08

[27] ISO29621:2010 Cosmetics-Microbiology-Guidelines for the Risk Assessment and Identification of Microbiologically Low-Risk Products.

Submit or recommend next manuscript to SCIRP and we will provide best service for you:

Accepting pre-submission inquiries through Email, Facebook, LinkedIn, Twitter, etc. A wide selection of journals (inclusive of 9 subjects, more than 200 journals)

Providing 24-hour high-quality service

User-friendly online submission system

Fair and swift peer-review system

Efficient typesetting and proofreading procedure

Display of the result of downloads and visits, as well as the number of cited articles

Maximum dissemination of your research work

Submit your manuscript at: http://papersubmission.scirp.org/

Or contact jcdsa@scirp.org 